\title{
Temperature-dependent genetics of thermotolerance between yeast species
}

1 Melanie B. Abrams ${ }^{1}$, Rachel B. Brem ${ }^{1 *}$,

$2 \quad{ }^{1}$ Brem Laboratory, UC Berkeley, Department of Plant and Microbial Biology, Berkeley, CA, USA

3 * Correspondence:

4 Corresponding Author

5 rbrem@berkeley.edu

6 Keywords: Saccharomyces, Ancient, Evolution, Thermotolerance, Cline, Adaptation

\section{Abstract}

8 Many traits of industrial and basic biological interest arose long ago, and manifest now as fixed differences between a focal species and its reproductively isolated relatives. In these systems, extant individuals can hold clues to the mechanisms by which phenotypes evolved in their ancestors. We harnessed yeast thermotolerance as a test case for such molecular-genetic inferences. In viability experiments, we showed that extant Saccharomyces cerevisiae survived at temperatures where cultures of its sister species $S$. paradoxus died out. Then, focusing on loci that contribute to this difference, we found that the genetic mechanisms of high-temperature growth changed with temperature. We also uncovered an enrichment of low-frequency variants at thermotolerance loci in S. cerevisiae population sequences, suggestive of a history of non-neutral selective forces acting at these genes. We interpret our results in light of a model of gradual acquisition of thermotolerance in the $S$. cerevisiae lineage by positive selection along a temperature cline. We propose that in an ancestral $S$. cerevisiae population, alleles conferring defects at a given temperature would have been resolved by adaptive mutations, expanding the range and setting the stage for further temperature advances. Together, our results and interpretation underscore the power of genetic approaches to explore how an ancient trait came to be.

\section{Introduction}

A central goal of evolutionary genetics is to understand how nature builds new phenotypes. Thanks to advances in statistical genetics and experimental evolution, mechanisms of trait evolution over relatively short timescales have come well within reach in the modern literature. By contrast, longerterm innovations have posed a more profound challenge for the field (Orr, 2001). In principle, for a phenotype that originated long ago and manifests now as a fixed difference between species, evolution could have refined the character along the entire divergence time of the respective taxa. In landmark cases, candidate-gene studies have shed light on suites of mutational changes of this kind between species, at a given model locus. This includes the order by which adaptive alleles were likely acquired, and/or the functional pressures that drove them (Bridgham et al., 2009; Baldwin et al., 2014; Dong et al., 2014; Sayou et al., 2014; Anderson et al., 2015; Daugherty et al., 2016; Sulak et al., 2016; Liu et al., 2018; Starr et al., 2018; Xie et al., 2018; Siddiq and Thornton, 2019; Pillai et al., 2020; Prieto-Godino et al., 2021). But, in most cases, any factor pursued by such a candidategene approach only represents part of the complex genetic architecture of an ancient trait. We still know relatively little about how evolution coordinates multiple adaptive loci over deep divergences. 
In the search for evolutionary principles, genetically tractable model systems can be of great utility. Saccharomyces yeasts are well suited for this purpose, and environmental yeast isolates have been studied extensively for their innovations within and between species (Hittinger, 2013). Thermotolerance is of particular interest because it tracks with phylogeny across the 20 million years of the Saccharomyces radiation (Gonçalves et al., 2011; Salvadó et al., 2011). Even the two most recent branches of the phylogeny exhibit a robust difference in this phenotype: $S$. cerevisiae acquired the ability to grow at temperatures near $40^{\circ} \mathrm{C}$ in the five million years since it diverged from its sister species, S. paradoxus (Sweeney et al., 2004; Gonçalves et al., 2011; Salvadó et al., 2011; Williams et al., 2015). Previously, a whole-genome mapping scheme was used to identify housekeeping genes at which variation between $S$. cerevisiae and $S$. paradoxus impacts growth at the high end of the $S$. cerevisiae temperature range (Weiss et al., 2018). These loci exhibit striking sequence differences between the species, and conservation in S. cerevisiae, consistent with a history of positive selection on pro-thermotolerance alleles (Weiss et al., 2018; Abrams et al., 2021a, 2021b). However, we have as yet little insight into the ecological dynamics by which this model trait evolved along the $S$. cerevisiae lineage.

Cases of adaptation across temperature clines are a mainstay of the evolutionary genetics literature (Turner et al., 2008; Prasad et al., 2011; Mimura et al., 2013; Savolainen et al., 2013; Robin et al., 2017; Dudaniec et al., 2018; Key et al., 2018; Endler, 2020; Tepolt and Palumbi, 2020; Calfee et al., 2021; Machado et al., 2021). Here we sought to explore the relevance of such a mechanism to the events by which $S$. cerevisiae gained its maximal thermotolerance phenotype. Given that we have no access to genotypes representing ancient intermediates between this species and $S$. paradoxus, we designed a strategy to interrogate the genetics of extant strains, focusing on contributing genes of major effect. We surveyed gene-environment interactions by these thermotolerance loci across warm temperatures, complementing previous studies of interspecies variation at a single high temperature (Weiss et al., 2018; Abrams et al., 2021b). And we investigated the frequency of variants at these loci with a population-genomic approach.

\section{$66 \quad 2.1$ Dose response growth assay}

67 For growth measurements in Figure 2, we assayed S. paradoxus Z1, S. cerevisiae DBVPG1373, and 68 S. cerevisiae DBVPG1373 with the S. paradoxus Z1 allele of ESP1, MYO1, AFG2, or CEP3 swapped in at the endogenous locus from (Weiss et al., 2018) (Table S2) as follows. Each strain was streaked from a $-80^{\circ} \mathrm{C}$ freezer stock onto a yeast peptone dextrose (YPD) agar plate and incubated at room temperature for 3 days. For each biological replicate, a single colony was inoculated into $5 \mathrm{~mL}$ liquid YPD and grown for 24 hours at $28^{\circ} \mathrm{C}$ with shaking at $200 \mathrm{rpm}$ to generate pre-cultures. Each pre-culture was backdiluted into $\mathrm{YPD}$ at an $\mathrm{OD}_{600} / \mathrm{mL}$ of 0.05 and grown for an additional 5.5-6 hours at $28^{\circ} \mathrm{C}$, shaking at $200 \mathrm{rpm}$, until reaching logarithmic phase. Each pre-culture was again back-diluted into $10 \mathrm{~mL}$ YPD in 1-inch diameter glass tubes with a target $\mathrm{OD}_{600} / \mathrm{mL}$ of 0.05 ; the actual $\mathrm{OD}_{600} / \mathrm{mL}$ of each was measured, after which it was grown at a temperature of interest $\left(28^{\circ} \mathrm{C}\right.$ or $35-38^{\circ} \mathrm{C}$ ) with shaking at 200rpm for 24 hours, and $\mathrm{OD}_{600} / \mathrm{mL}$ was measured again. The growth efficiency for each replicate was calculated as the difference between these final and initial $\mathrm{OD}_{600} / \mathrm{mL}$ values. We used the growth efficiency from all days and all temperatures of a given strain $\mathrm{s}$ as input into a two-factor type 2 ANOVA test for a temperature-by-strain effect comparing s with $S$. 81 cerevisiae. 
82 For growth measurements of ESP1 swap strains with different donors, as reported in Figure S2,

83 cultures were grown and measured as above, except that the only temperature was $36^{\circ} \mathrm{C}$.

\section{$84 \quad 2.2 \quad$ Viability assay}

85 For the survey of viability phenotypes at high temperatures across wild-type isolates in Figure 2, 86 strains were streaked out and a colony of each was pre-cultured in liquid as for $39^{\circ} \mathrm{C}$ growth above, 87 except that the initial pre-culture to achieve saturation lasted 48 hours. Each pre-culture was back88 diluted into $10 \mathrm{~mL}$ of YPD to reach an $\mathrm{OD}_{600} / \mathrm{mL}$ of 0.05 and then cultured for 24 hours at the temperature of interest $\left(28^{\circ} \mathrm{C}-39^{\circ} \mathrm{C}\right)$. The viability of both the precultures and the cultures after 24 hours at the temperature of interest were measured with a spotting assay, where we diluted aliquots from the culture in a 1:10 series and spotting $3 \mu \mathrm{L}$ of each dilution for growth on a solid YPD plate. After incubation at $28^{\circ} \mathrm{C}$ for two days, we used the dilution corresponding to the densest spot that was not a lawn for to determine viability: we counted the number of colonies in each of the two technical replicate spots, formulated the number of colony forming units per mL of undiluted culture $(\mathrm{CFU} / \mathrm{mL})$. We determined the change in viable cells by subtracting the number of cells in the culture at the initial time point from that at the final timepoint, based on the CFU/mL count and the culture volume. We evaluated the significance of the difference between $S$. cerevisiae and $S$. paradoxus at a given temperature using a one-sided Mann-Whitney U test.

\subsection{Tajima's D in Wine/European S. cerevisiae}

100 Tajima's D tabulates the difference between the average number of differences in pairs of sequences in a population sample and the number of variant sites in the sample. When the latter is of much

102

103 bigger magnitude and $\mathrm{D}$ is negative, it indicates that variation in the sample is accounted for mostly by rare alleles. This pattern is expected some time after a selective sweep when de novo mutations arise on the swept haplotype; it can also reflect weak purifying selection or a history of population expansion (Suzuki, 2010). Taking as input the VCFs reporting inheritance in the strains of a given $S$. cerevisiae population from (Peter et al., 2018), we used VCF-kit (Cook and Andersen, 2017) to calculate Tajima's D for the region from coding start to coding stop for each gene. We then developed a resampling test to assess enrichment trends in Tajima's D in genes of interest against a genomic null. This scheme normalizes out impacts on Tajima's D values from events which affect the whole genome, such as a population expansion; that said, formally, any results from such an empirical outlier-based analysis serve as suggestive rather than conclusive evidence for non-neutral evolution (Teshima et al., 2006; Thornton and Jensen, 2007). For the test, we sampled 10,000 random cohorts of genes from the genome with the same number of essential and nonessential genes as our thermotolerance cohort (Winzeler et al., 1999), and we used as an empirical P-value the proportion of random cohorts where the median Tajima's D was less than or equal to that of our thermotolerance cohort. Application of this test to the Wine/European S. cerevisiae population (362 strains) is reported in Figure 3 of the main text. Applied to the Mosaic Region 3 (113 strains), Mixed

118 Origin (72 strains), Sake (47 strains), and Brazilian Bioethanol (35 strains), the four next most deeply 119 sampled populations from (Peter et al., 2018), this test for enrichment of low Tajima's D among our 120 four focal thermotolerance genes yielded $P=0.8351,0.975,0.8624$, and 0.0348 respectively. 


\section{Results}

122 For an initial study of the genetics of yeast species variation in temperature response, we chose to

123 harness DBVPG1373, an S. cerevisiae isolate from Dutch soil, and Z1, an S. paradoxus isolate from an oak tree in England. We anticipated that detailed analyses using these strains, as representatives of their respective species, could accelerate the discovery of more general principles (Weiss et al., 2018; Abrams et al., 2021a, 2021b). We developed an assay quantifying cell viability in a given liquid culture before and after incubation at a temperature of interest, and we implemented this approach for each species in turn. The results revealed an advantage for $S$. cerevisiae over $S$. paradoxus at temperatures above $35^{\circ} \mathrm{C}$ (Figure 1), consistent with previous growth-based surveys (Sweeney et al., 2004; Gonçalves et al., 2011; Salvadó et al., 2011; Williams et al., 2015). S. cerevisiae maintained viability at all temperatures tested, whereas by $37^{\circ} \mathrm{C}, S$. paradoxus became inviable, with no evidence for spontaneous rescue even over long incubation times (Figure 1). Using the latter as a window onto the phenotype of the common ancestor of $S$. cerevisiae and $S$. paradoxus, we would envision that the latter ancient population would have gone extinct rather than adapting, if exposed to temperatures at the high end of the range tolerated by modern $S$. cerevisiae.

We next aimed to investigate the genetics of temperature response as it differs between extant $S$. cerevisiae and $S$. paradoxus, to motivate inferences about the evolution of the maximal thermotolerance trait. We focused on four genes - the cell division factors ESP1, MYO1, and CEP3, and the ribosome maturation factor $A F G 2$-where alleles from modern $S$. paradoxus compromise growth at $39^{\circ} \mathrm{C}$ (Weiss et al., 2018). We made use of strains of the $S$. cerevisiae DBVPG1373 background harboring the allele of each gene in turn from $S$. paradoxus Z1. In each, we measured the growth phenotype as a dose-response across temperatures, and observed a drop as temperature increased (Figure 2). In the $S$. cerevisiae background, $S$. paradoxus $\mathrm{Z} 1$ alleles eroded growth efficiency at temperatures well below the hard limit of viability for the $\mathrm{Z} 1$ wild-type $\left(\sim 38^{\circ} \mathrm{C}\right)$, an effect that reached significance for three of our four focal genes (Figure 2). We conclude that many problems posed by $S$. paradoxus $\mathrm{Z} 1$ alleles at the high end of our temperature range also manifest to a lesser extent at lower temperatures.

148 Inspecting the shape of the temperature dose-responses of allelic effects, we noted quantitative differences between our loci. At the chromatid separase gene ESP1, the Z1 allele conferred an appreciable drop in growth efficiency at $36^{\circ} \mathrm{C}$ and supported almost no growth by $37^{\circ} \mathrm{C}$ (Figure $2 \mathrm{~B}$ ). By contrast, at $A F G 2$, the $S$. paradoxus $\mathrm{Z} 1$ allele was sufficient for growth approximating that of $S$. cerevisiae until $38^{\circ} \mathrm{C}$ (Figure 2D). The dose-response of allelic effects at $M Y O 1$, encoding a class II myosin heavy chain, lay between these two extremes (Figure 2C). This differential susceptibility to temperature across the genes of our set likely reflects distinguishing properties of their structure and function, and of the interspecies variants they harbor.

156 We reasoned that trends from our temperature dose-response approach would be most informative when they were conserved across $S$. paradoxus as a species. To pursue this, we earmarked ESPI, whose $S$. paradoxus $\mathrm{Z} 1$ allele had exhibited the sharpest falloff with temperature among the genes of our set (Figure 2B). We repeated our growth efficiency experiments in strains of S. cerevisiae DBVPG1373 harboring ESP1 from other $S$. paradoxus donors beside the Z1 strain. These transgenics, which phenocopy wild-type DBVPG1373 at $28^{\circ} \mathrm{C}$ (Weiss et al., 2018), all dropped off in growth efficiency by $36^{\circ} \mathrm{C}$ (Figure S2), as expected if the temperature preference of ESP1 were

163 ancestral to, and shared across, extant $S$. paradoxus populations.

164 Together, these dose-response results make clear that the functions of $S$. paradoxus alleles at our 165 focal genes break down at distinct temperatures between $35^{\circ} \mathrm{C}$ and $38^{\circ} \mathrm{C}$ - suggesting similar gene- 
by-environment effects in the ancestor of $S$. cerevisiae and $S$. paradoxus, if it sampled a range of temperature conditions over evolutionary history.

168 To gain insight into past and current selective pressures on thermotolerance loci, we turned to a molecular-evolution approach. Previous work on Saccharomyces thermotolerance genes has emphasized sequence divergence between species (Weiss et al., 2018; Abrams et al., 2021a, 2021b). For a complementary focus on population variation within $S$. cerevisiae, we analyzed the Tajima's D statistic, which formulates properties of sequence variants in a population into a test of how well the sequence fits the expectations under a neutral evolutionary model (Tajima, 1989; Biswas and Akey, 2006). We first developed a resampling-based scheme that compares Tajima's D between a gene cohort of interest and a genomic null. This enables a non-parametric assessment of significance and normalizes out potential effects of demography on the statistic (see Methods). Expecting that our test would have maximal power in a data set of large sample size, we focused on the most deeplysampled S. cerevisiae population in current compendia, comprising isolates from vineyards and European soil (Peter et al., 2018). Examining our four focal thermotolerance genes, we detected an enrichment for low, negative Tajima's D at these loci across S. cerevisiae genomes (Figure 3 and Table S1), reporting an excess of rare variants-as expected after a selective sweep, or under constraints from purifying selection (Biswas and Akey, 2006; Suzuki, 2010). We repeated this analysis using more comprehensive sets of hits from interspecies thermotolerance screens (Weiss et al., 2018; Abrams et al., 2021a, 2021b), and detected strong signal for low, negative Tajima's D at these loci in vineyard/European $S$. cerevisiae in every case (Table S1). Interestingly, ESP1 exhibited the most negative Tajima's D value among all thermotolerance genes (Table S1), dovetailing with the strong effect of variation at this gene in phenotypic analyses (Weiss et al. 2018 and Figure 2). The trend for low, negative Tajima's D in thermotolerance genes was detectable but not consistent across other shallowly-sampled populations of $S$. cerevisiae (see Methods), potentially reflecting weaker power or weaker selection in the latter relative to vineyard/European strains. In either case, our data establish that thermotolerance gene variation in some modern $S$. cerevisiae populations is consistent with a history of non-neutral evolution.

\section{Discussion}

Most trait differences that define long-diverged species are likely the product of suites of unlinked variants that have come together over long timescales. Tracing the ancient evolutionary events at such loci remains a central challenge in the field. In this work, we have characterized thermotolerance genes in extant yeast strains and species to inform models of the evolution of the trait in S. cerevisiae.

199 Our data have shown that temperatures below the high end of the $S$. cerevisiae range are lethal for $S$. paradoxus. This complements previous surveys of the Saccharomyces clade using growth-based assays (Sweeney et al., 2004; Gonçalves et al., 2011; Salvadó et al., 2011) and reveals that, under high temperature conditions, a given culture of $S$. paradoxus will die off rather than adapting. Assuming similar behavior in an ancestor of $S$. cerevisiae before the rise of its modern thermotolerance profile, we infer that the latter event was unlikely to be precipitated by a single jump to a hot growth environment, long ago in history. Rather, we favor the hypothesis that thermotolerance evolution through the $S$. cerevisiae lineage proceeded along a temperature cline, as has been documented in elegant local-adaptation case studies (Mimura et al., 2013; Robin et al., 2017; Dudaniec et al., 2018; Key et al., 2018; Tepolt and Palumbi, 2020). In this scenario, a welladapted ancestral $S$. cerevisiae population in a given temperature niche would have acquired variants that resolved defects manifesting in slightly warmer conditions, and expanded its range. If the $S$. cerevisiae lineage did go through a series of such transitions in acquiring maximal thermotolerance, 
212 it would echo the principle that gradual exposure to increasing stresses fosters adaptation and reduces

213 the risk of extinction, relative to a sudden, high dose of stress (Lindsey et al., 2013).

214 We have also leveraged results from genetic mapping of S. cerevisiae thermotolerance to trace the

215 temperature dependence of contributing genes. This contrasts with approaches that search genomes

216 for variants associated with environment or population variables (Rellstab et al., 2015; Hoban et al.,

217

218

219

220

221

222

223

224

225

226

227 2016), in that we focus directly on bona fide causal determinants of the trait of interest. Our strategy has allowed us to discern differences between thermotolerance loci in terms of the temperatures at which alleles from modern $S$. paradoxus fail in their growth functions. In other words, the genetic mechanisms of growth change with temperature in this system. If such effects were at play in the $S$. cerevisiae ancestor, as it sampled conditions and niches during the acquisition of thermotolerance, each locus would have come under selective pressure at the temperature where its defect manifested. This phenomenon, by which the weakest point in the genetic network targeted by evolution changes as conditions change, has been termed "whack-a-mole" dynamics (Shin and MacCarthy, 2015). Ultimately, a complete model of adaptation across time and environment will take into account the shifts in the evolutionary landscape from this effect as well as from constant changes in genetic background (Starr and Thornton, 2016).

228

If the ancient $S$. cerevisiae population did adapt progressively along a temperature cline, what would the ecology have been? In principle, migrants from temperate physical locales could have advanced to warmer and warmer locales, perhaps terminating in the hot East Asian environments to which the ancestor of modern S. cerevisiae has been traced (Peter et al., 2018). Alternatively, the trait syndrome in this species - a unique ability to tolerate ethanol as well as heat, with both given off by fermentative metabolism - could have arisen as a specialization to kill off microbial competitors in nutrient-rich substrates, regardless of the endemic temperature of the location (Goddard, 2008; Salvadó et al., 2011). If so, variants would have been acquired, potentially over millions of generations, gradually to ratchet up fermentative activity and tolerance to its byproducts, with our genetic insights thus far largely restricted to the latter.

Our work also leaves open the dating of any such events. In several S. cerevisiae populations, we have uncovered an enrichment of rare variants at thermotolerance genes. This signature of nonpositively selected haplotype, or for negative selection to maintain a fitness-relevant haplotype that arose long ago. We favor the latter hypothesis, given that prior work across populations has also made clear that $S$. cerevisiae alleles at our focal genes are partly sufficient for maximal thermotolerance, conserved within the species, and divergent from S. paradoxus (Weiss et al., 2018; Abrams et al., 2021b). We thus propose that many thermotolerance alleles were acquired in ancient selective sweeps, before the radiation of modern $S$. cerevisiae, and have been maintained since then by purifying selection. That said, later refinements in particular populations of S. cerevisiae may also have strengthened beneficial facets of the trait, added regulatory tuning, or eliminated antagonistic pleiotropic "side effects" that were niche-specific. A comprehensive genetic and ecological reconstruction of this history may be out of our current grasp, especially in light of the caveats of our approach using a laboratory setting and extant strain backgrounds. Nonetheless, our data add compelling detail to an emerging consensus view of how evolution built maximal thermotolerance in S. cerevisiae.

\section{Conflict of Interest}

255 The authors declare that the research was conducted in the absence of any commercial or financial

256 relationships that could be construed as a potential conflict of interest. 


\section{Author Contributions}

258 M.A. and R.B.B. designed the study. MBA conducted experimental work and performed data

259 collection and analysis. MBA and RBB wrote the manuscript.

$260 \quad 7 \quad$ Funding

261 This work was supported by NSF GRFP DGE 1752814 to M.A. and NIH R01 GM120430 to R.B.B.

\section{Acknowledgments}

263 The authors thank Faisal AlZaben, Abel Duarte, and Jude Edwards for experimental support and

264 Adam Arkin for his generosity with computational resources.

\section{Data Availability Statement}

266 Strains and plasmids are available upon request. Custom scripts for the analysis will be available at https://github.com/melanieabrams-pub/.

\section{References}

269

270

271

272

273

274

275

276

277

278

279

280

281

282

283

284

285

286

287

288

289

290

Abrams, M. B., Chuong, J. N., AlZaben, F., Dubin, C. A., Skerker, J. M., and Brem, R. B. (2021a). Barcoded reciprocal hemizygosity analysis via sequencing illuminates the complex genetic basis of yeast thermotolerance. doi:10.1101/2021.07.26.453780.

Abrams, M. B., Dubin, C. A., AlZaben, F., Bravo, J., Joubert, P. M., Weiss, C. V., et al. (2021b). Population and comparative genetics of thermotolerance divergence between yeast species. G3 Genes|Genomes|Genetics. doi:https://doi.org/10.1093/g3journal/jkab139.

Anderson, D. W., McKeown, A. N., and Thornton, J. W. (2015). Intermolecular epistasis shaped the function and evolution of an ancient transcription factor and its DNA binding sites. eLife 4, e07864. doi:10.7554/eLife.07864.

Baldwin, M. W., Toda, Y., Nakagita, T., O’Connell, M. J., Klasing, K. C., Misaka, T., et al. (2014). Sensory biology. Evolution of sweet taste perception in hummingbirds by transformation of the ancestral umami receptor. Science 345, 929-933. doi:10.1126/science.1255097.

Biswas, S., and Akey, J. M. (2006). Genomic insights into positive selection. Trends in Genetics 22, 437-446. doi:10.1016/j.tig.2006.06.005.

Bridgham, J. T., Ortlund, E. A., and Thornton, J. W. (2009). An epistatic ratchet constrains the direction of glucocorticoid receptor evolution. Nature 461, 515-519. doi:10.1038/nature08249.

Calfee, E., Gates, D., Lorant, A., Perkins, M. T., Coop, G., and Ross-Ibarra, J. (2021). Selective sorting of ancestral introgression in maize and teosinte along an elevational cline. PLOS Genetics 17, e1009810. doi:10.1371/journal.pgen.1009810.

Cook, D. E., and Andersen, E. C. (2017). VCF-kit: assorted utilities for the variant call format. Bioinformatics 33, 1581-1582. doi:10.1093/bioinformatics/btx011. 
Daugherty, M. D., Schaller, A. M., Geballe, A. P., and Malik, H. S. (2016). Evolution-guided functional analyses reveal diverse antiviral specificities encoded by IFIT1 genes in mammals. eLife 5, e14228. doi:10.7554/eLife.14228.

Dong, S., Stam, R., Cano, L. M., Song, J., Sklenar, J., Yoshida, K., et al. (2014). Effector specialization in a lineage of the Irish potato famine pathogen. Science 343, 552-555. doi:10.1126/science. 1246300 .

Dudaniec, R. Y., Yong, C. J., Lancaster, L. T., Svensson, E. I., and Hansson, B. (2018). Signatures of local adaptation along environmental gradients in a range-expanding damselfly (Ischnura elegans). Molecular Ecology 27, 2576-2593. doi:10.1111/mec.14709.

Endler, J. A. (2020). Geographic Variation, Speciation and Clines. (MPB-10), Volume 10. Princeton University Press doi:10.2307/j.ctvx5wbdg.

Goddard, M. R. (2008). Quantifying the complexities of Saccharomyces cerevisiae's ecosystem engineering via fermentation. Ecology 89, 2077-2082. doi:10.1890/07-2060.1.

Gonçalves, P., Valério, E., Correia, C., Almeida, J. M. G. C. F. de, and Sampaio, J. P. (2011). Evidence for Divergent Evolution of Growth Temperature Preference in Sympatric Saccharomyces Species. PLOS ONE 6, e20739. doi:10.1371/journal.pone.0020739.

Hittinger, C. T. (2013). Saccharomyces diversity and evolution: a budding model genus. Trends Genet 29, 309-317. doi:10.1016/j.tig.2013.01.002.

Hoban, S., Kelley, J. L., Lotterhos, K. E., Antolin, M. F., Bradburd, G., Lowry, D. B., et al. (2016). Finding the Genomic Basis of Local Adaptation: Pitfalls, Practical Solutions, and Future Directions. Am Nat 188, 379-397. doi:10.1086/688018.

Key, F. M., Abdul-Aziz, M. A., Mundry, R., Peter, B. M., Sekar, A., D’Amato, M., et al. (2018). Human local adaptation of the TRPM8 cold receptor along a latitudinal cline. PLOS Genetics 14, e1007298. doi:10.1371/journal.pgen.1007298.

Lindsey, H. A., Gallie, J., Taylor, S., and Kerr, B. (2013). Evolutionary rescue from extinction is contingent on a lower rate of environmental change. Nature 494, 463-467. doi:10.1038/nature11879.

Liu, Q., Onal, P., Datta, R. R., Rogers, J. M., Schmidt-Ott, U., Bulyk, M. L., et al. (2018). Ancient mechanisms for the evolution of the bicoid homeodomain's function in fly development. Elife 7. doi:10.7554/eLife.34594.

Machado, H. E., Bergland, A. O., Taylor, R., Tilk, S., Behrman, E., Dyer, K., et al. (2021). Broad geographic sampling reveals the shared basis and environmental correlates of seasonal adaptation in Drosophila. eLife 10, e67577. doi:10.7554/eLife.67577.

Mimura, M., Ono, K., Goka, K., and Hara, T. (2013). Standing variation boosted by multiple sources of introduction contributes to the success of the introduced species, Lotus corniculatus. Biol Invasions 15, 2743-2754. doi:10.1007/s10530-013-0488-x. 
Orr, H. A. (2001). The genetics of species differences. Trends Ecol Evol 16, 343-350. doi:10.1016/s0169-5347(01)02167-x.

Peter, J., Chiara, M. D., Friedrich, A., Yue, J.-X., Pflieger, D., Bergström, A., et al. (2018). Genome evolution across 1,011 Saccharomyces cerevisiae isolates. Nature 556, 339-344. doi:10.1038/s41586-018-0030-5.

Pillai, A. S., Chandler, S. A., Liu, Y., Signore, A. V., Cortez-Romero, C. R., Benesch, J. L. P., et al. (2020). Origin of complexity in haemoglobin evolution. Nature 581, 480-485. doi:10.1038/s41586-020-2292-y.

Prasad, A., Croydon-Sugarman, M. J. F., Murray, R. L., and Cutter, A. D. (2011). TemperatureDependent Fecundity Associates with Latitude in Caenorhabditis Briggsae. Evolution 65, 52 63. doi:10.1111/j.1558-5646.2010.01110.x.

Prieto-Godino, L. L., Schmidt, H. R., and Benton, R. (2021). Molecular reconstruction of recurrent evolutionary switching in olfactory receptor specificity. eLife 10, e69732. doi:10.7554/eLife.69732.

Rellstab, C., Gugerli, F., Eckert, A. J., Hancock, A. M., and Holderegger, R. (2015). A practical guide to environmental association analysis in landscape genomics. Mol Ecol 24, 4348-4370. doi: $10.1111 / \mathrm{mec} .13322$.

Robin, C., Andanson, A., Saint-Jean, G., Fabreguettes, O., and Dutech, C. (2017). What was old is new again: thermal adaptation within clonal lineages during range expansion in a fungal pathogen. Molecular Ecology 26, 1952-1963. doi:10.1111/mec.14039.

Salvadó, Z., Arroyo-López, F. N., Guillamón, J. M., Salazar, G., Querol, A., and Barrio, E. (2011). Temperature adaptation markedly determines evolution within the genus Saccharomyces. Appl Environ Microbiol 77, 2292-2302. doi:10.1128/AEM.01861-10.

Savolainen, O., Lascoux, M., and Merilä, J. (2013). Ecological genomics of local adaptation. Nature Reviews Genetics 14, 807-820. doi:10.1038/nrg3522.

Sayou, C., Monniaux, M., Nanao, M. H., Moyroud, E., Brockington, S. F., Thévenon, E., et al. (2014). A Promiscuous Intermediate Underlies the Evolution of LEAFY DNA Binding Specificity. Science 343, 645-648. doi:10.1126/science.1248229.

Shin, J., and MacCarthy, T. (2015). Antagonistic Coevolution Drives Whack-a-Mole Sensitivity in Gene Regulatory Networks. PLoS Comput Biol 11, e1004432. doi:10.1371/journal.pcbi.1004432.

Siddiq, M. A., and Thornton, J. W. (2019). Fitness effects but no temperature-mediated balancing selection at the polymorphic Adh gene of Drosophila melanogaster. PNAS 116, 21634-21640. doi:10.1073/pnas.1909216116.

Starr, T. N., Flynn, J. M., Mishra, P., Bolon, D. N. A., and Thornton, J. W. (2018). Pervasive contingency and entrenchment in a billion years of Hsp90 evolution. PNAS 115, 4453-4458. doi:10.1073/pnas.1718133115. 
364

365

366

367

368

369

370

371

372

373

374

375

376

377

378

379

380

381

382

383

384

385

386

387

388

389

390

391

392

393

394

395

396

Starr, T. N., and Thornton, J. W. (2016). Epistasis in protein evolution. Protein Science 25, 1204 1218. doi:10.1002/pro.2897.

Sulak, M., Fong, L., Mika, K., Chigurupati, S., Yon, L., Mongan, N. P., et al. (2016). TP53 copy number expansion is associated with the evolution of increased body size and an enhanced DNA damage response in elephants. eLife 5, e11994. doi:10.7554/eLife.11994.

Suzuki, Y. (2010). Statistical methods for detecting natural selection from genomic data. Genes Genet Syst 85, 359-376. doi:10.1266/ggs.85.359.

Sweeney, J. Y., Kuehne, H. A., and Sniegowski, P. D. (2004). Sympatric natural Saccharomyces cerevisiae and S. paradoxus populations have different thermal growth profiles. FEMS Yeast Res. 4, 521-525. doi:10.1016/S1567-1356(03)00171-5.

Tajima, F. (1989). Statistical Method for Testing the Neutral Mutation Hypothesis by DNA Polymorphism. Genetics 123, 585-595.

Tepolt, C. K., and Palumbi, S. R. (2020). Rapid Adaptation to Temperature via a Potential Genomic Island of Divergence in the Invasive Green Crab, Carcinus maenas. Frontiers in Ecology and Evolution 8, 411. doi:10.3389/fevo.2020.580701.

Teshima, K. M., Coop, G., and Przeworski, M. (2006). How reliable are empirical genomic scans for selective sweeps? Genome Res 16, 702-712. doi:10.1101/gr.5105206.

Thornton, K. R., and Jensen, J. D. (2007). Controlling the False-Positive Rate in Multilocus Genome Scans for Selection. Genetics 175, 737-750. doi:10.1534/genetics.106.064642.

Turner, T. L., Levine, M. T., Eckert, M. L., and Begun, D. J. (2008). Genomic Analysis of Adaptive Differentiation in Drosophila melanogaster. Genetics 179, 455-473. doi:10.1534/genetics.107.083659.

Weiss, C. V., Roop, J. I., Hackley, R. K., Chuong, J. N., Grigoriev, I. V., Arkin, A. P., et al. (2018). Genetic dissection of interspecific differences in yeast thermotolerance. Nat Genet 50, 15011504. doi:10.1038/s41588-018-0243-4.

Williams, K. M., Liu, P., and Fay, J. C. (2015). Evolution of ecological dominance of yeast species in high-sugar environments. Evolution 69, 2079-2093. doi:10.1111/evo.12707.

Winzeler, E. A., Shoemaker, D. D., Astromoff, A., Liang, H., Anderson, K., Andre, B., et al. (1999). Functional Characterization of the S. cerevisiae Genome by Gene Deletion and Parallel Analysis. Science 285, 901-906. doi:10.1126/science.285.5429.901.

Xie, J., Li, Y., Shen, X., Goh, G., Zhu, Y., Cui, J., et al. (2018). Dampened STING-Dependent Interferon Activation in Bats. Cell Host \& Microbe 23, 297-301.e4. doi:10.1016/j.chom.2018.01.006. 


\section{Figure Captions}

$398 \quad$ 11.1 Figure 1. S. paradoxus is inviable at high temperature.

399 Each column reports the viability of a culture of $S$. cerevisiae DBVPG1373 or S. paradoxus Z1 at the

400 indicated temperature. The $y$-axis reports the number of viable colony forming units (CFU) per unit

401 of optical density after 24 hours of incubation, as a difference from the analogous quantity at time

402 zero. Each point reports results from one biological replicate, and each bar height reports the average 403 across replicates $(n=3-6)$. * $P<0.05$ for a one-sided Mann-Whitney $U$ test assessing the advantage

404 of $S$. cerevisiae over $S$. paradoxus at a given temperature.

\subsection{Figure 2. S. paradoxus alleles of thermotolerance genes confer temperature-dependent defects in the $S$. cerevisiae background.}

407 Each panel reports the results of growth experiments of a strain of S. cerevisiae DBVPG1373

408 harboring the indicated gene from $S$. paradoxus Z1, or the respective wild-type parent strains (WT), across a temperature gradient. In a given panel, the $y$-axis reports growth efficiency, the optical density reached by the culture after 24 hours at the temperature indicated on the $x$-axis, as a difference from the analogous quantity at time zero. Each point reports results from one biological replicate $(n=3)$, and the line represents the average growth efficiency of the indicated strain across the temperature gradient. ${ }^{*}, P \square<\square 10^{-3}$ for the strain by temperature interaction term of a two-factor ANOVA, in a comparison between the indicated strain and wild-type $S$. cerevisiae.

\subsection{Figure 3. Thermotolerance genes are enriched for low Tajima's D in S. cerevisiae.}

416 The $x$-axis reports the median Tajima's D across a gene set of interest in genomes of wine/European 417 strains of $S$. cerevisiae from (Peter et al., 2018). Blue bars reflect the results from genomic

418 resampling, with the $y$-axis reporting the number of randomly chosen gene sets with the median 419 Tajima's D shown on the $x$, and the blue curve showing a kernel density estimate of the histogram bar values. The red vertical line reports the Tajima's D value of the four thermotolerance genes characterized in Figure 2, corresponding to resampling $P=0.0263$. 


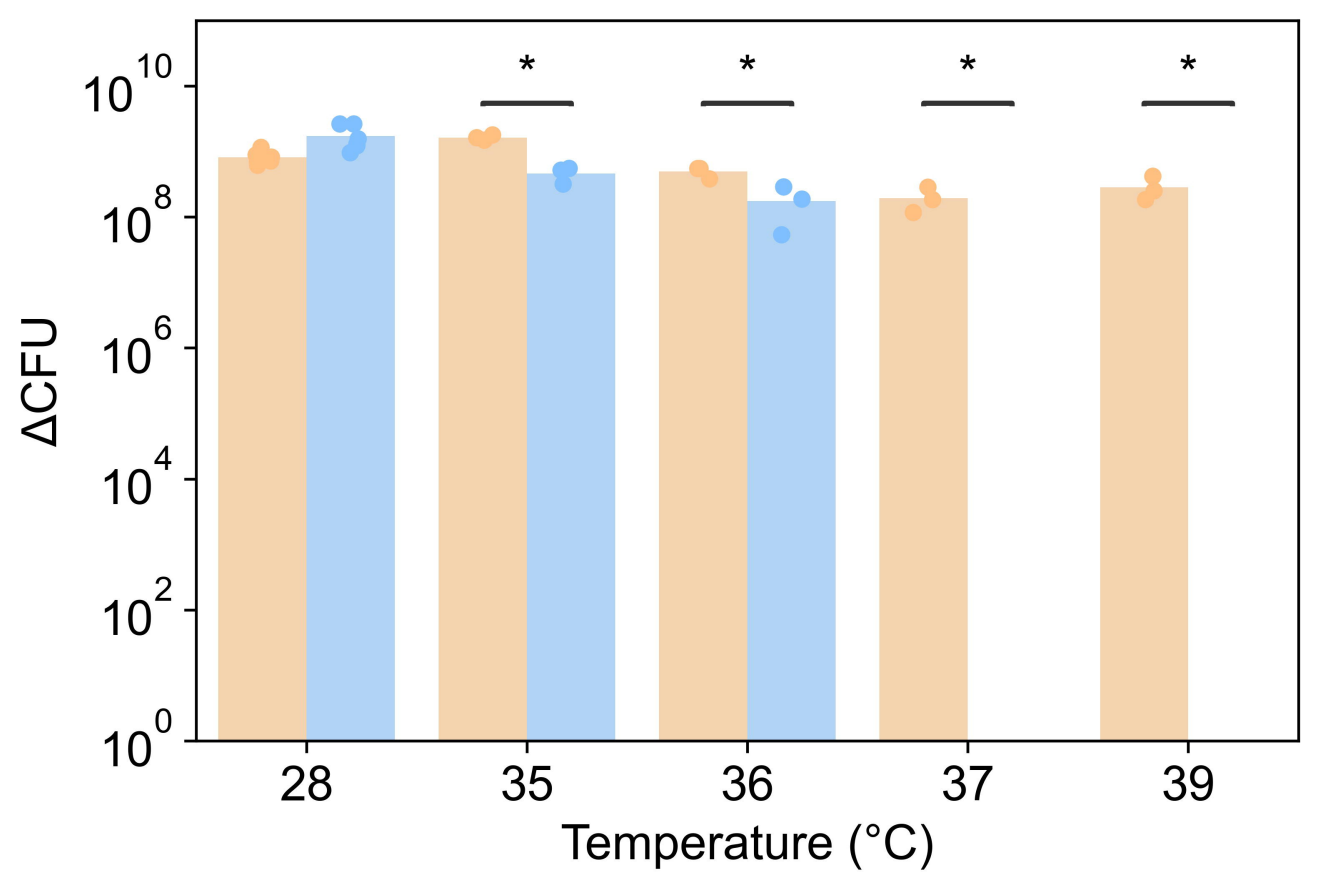

Sc 

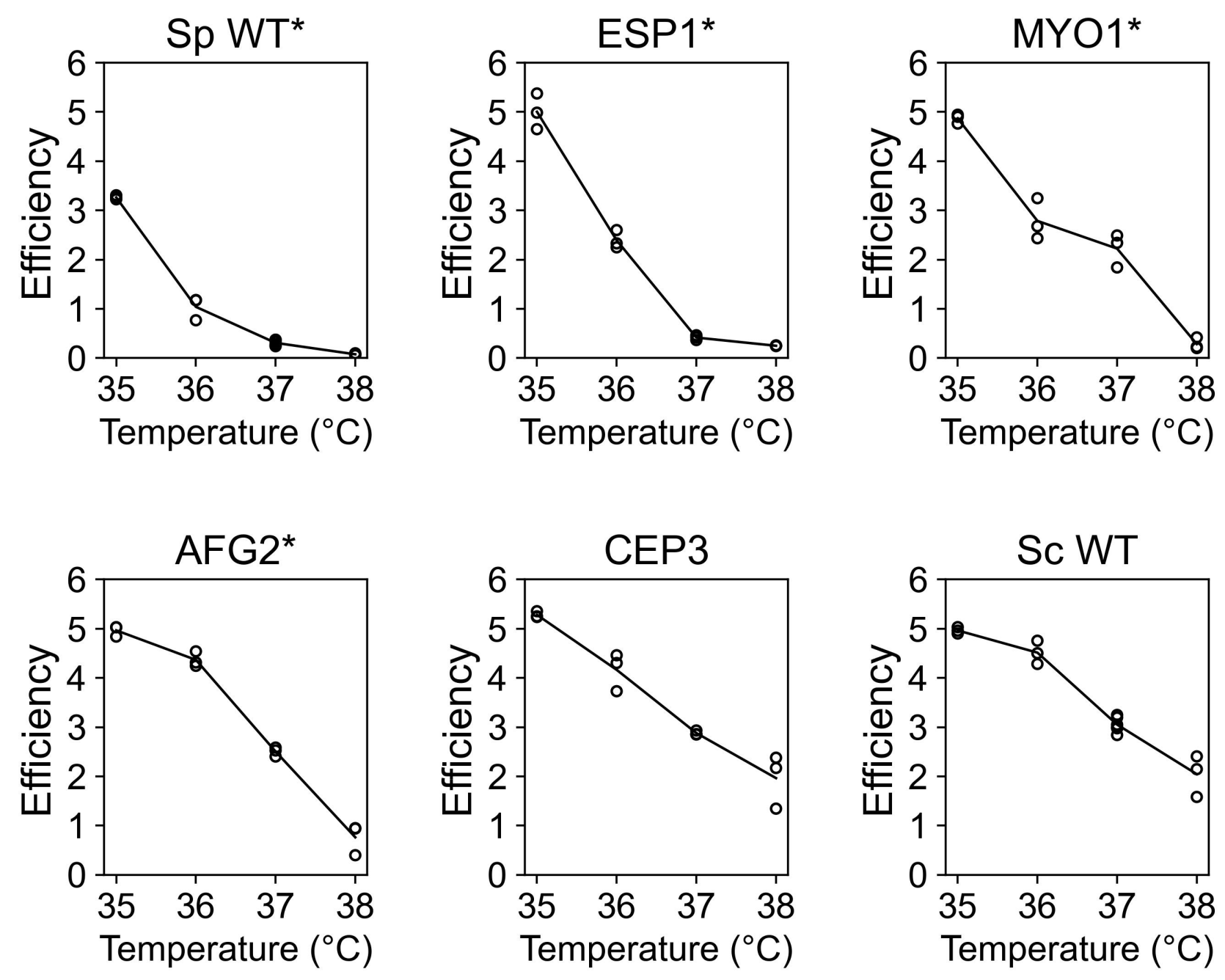


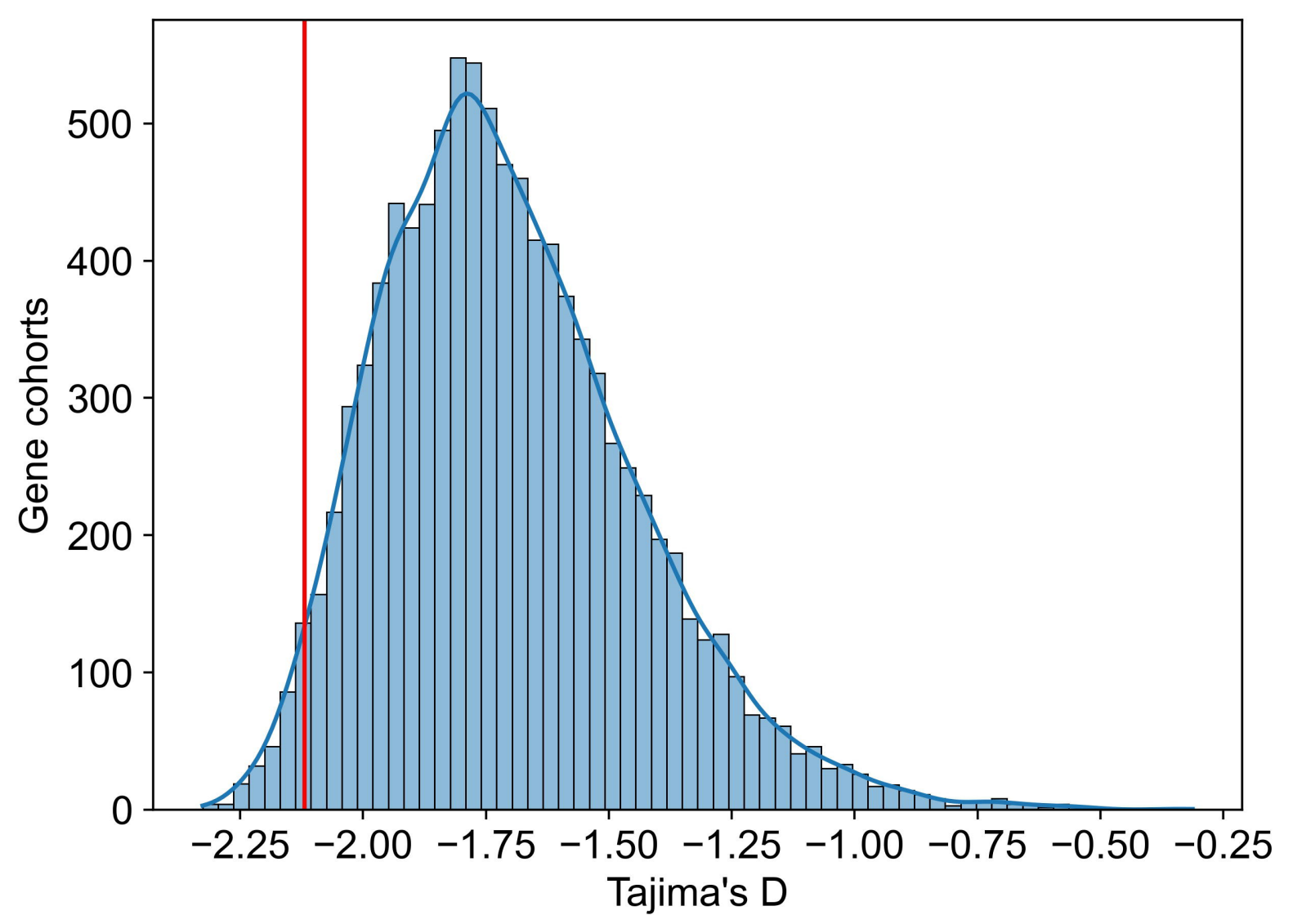

